\title{
Prepuce health and childhood circumcision: Choices in Canada
}

\author{
Emmanuel 0. Abara, MD
}

Clinical Sciences Division, Northern Ontario Medical School, Sudbury/Thunder Bay, ON, Canada

Cite as: Can Urol Assoc J 2017;11(1-2Suppl1):S55-62. http://dx.doi.org/10.5489/cuaj.4447

judicious choices in a culturally sensitive environment will offer the Canadian neonate desirable care of the prepuce for life.

See related commentary on page $\mathbf{S 6 3 .}$

\section{Abstract}

Introduction: Worldwide, almost $100 \%$ of boys are born with penises with a "hood" called prepuce or foreskin. In the course of the boy's life, the prepuce can be circumcised, can become affected by diseased (e.g., phimosis), or a can become infected and hurt the neonate (and his sexual partner) in adulthood. The objectives of this report are to: 1) review the state, function, fate, and care of the prepuce in childhood, with focus on the neonate, in Canada; 2) understand the current practice of childhood male circumcision in terms of age, indications, performers, techniques, outcomes, and education; and 3) consider ways to sustain a good healthcare professional-parental dialogue for safe practices that are accessible, acceptable, and culturally sensitive in the care of the prepuce.

Methods: A literature review was carried out in the English language through the major databases: PubMed (MEDLINE), EMBASE, the Cochrane Library, CINAHL, Web of Science (WOS) Core Collection, LILAC, WHO/UNAIDS, Clinical Trials (www.clinic altrials.gov), Google Scholar, and grey literature. Search words included: prepuce, diseases of prepuce, prepuce in the neonate, prepuce in the neonate in Canada, male circumcision, childhood male circumcision, neonatal circumcision, neonatal circumcision in Canada, complications of neonatal circumcision in Canada, and circumcision adverse events.

Results: From 1970-1999, three of 10 Canadian newborn males were circumcised for religious, cultural, and medical reasons. The rest of the neonates, if alive, are living with their prepuce; $<4 \%$ expected to require treatment for afflictions of the prepuce at some point. There are several providers of circumcision with different levels of training and competencies and using a diversity of devices and techniques. Neonatal and childhood circumcision in Canada is carried out to fulfill parental wishes, as well as for medical, religious, and cultural reasons. Appropriate informed consent and education regarding choices of care of the neonatal prepuce and genitals are vital.

Conclusions: Going by current prevalent rate of circumcision in Canada, most Canadian newborn males are likely to live out their lives with an intact prepuce. Despite the age-old debate, childhood circumcision is likely to remain. There is need for careful and proper discussion of the potential risks and benefits, including alternatives, costs, and personal/psychological factors. Acceptance, access, and

\section{Introduction}

Worldwide, close to $100 \%$ of boys are born with a phallus with a "hood" known as the prepuce or foreskin. The few born without a full prepuce may have congenital anomalies, such as hypospadias. The prepuce can be circumcised, can become affected by disease (e.g., phimosis), or a can become infected and hurt the neonate (and his sexual partner) in adulthood. In Canada, the fate of the prepuce often rests upon the choices of the parents of the neonate, who are influenced by personal values intertwined in religious and cultural beliefs, costs of care, guidance from healthcare professionals, and current healthcare policy.

Proper care of a penis with intact prepuce is simple and helps prevent bothersome ailments in life. Several authors ${ }^{1-4}$ have advocated this, often providing guidelines and time schedules for different interventions, if required. Circumcision, as a choice of care, has been practiced for over 5000 years; despite the challenges and arguments against and for, it is currently practiced in all the provinces and territories of Canada at varying prevalence rates.

The objectives of this review are to: 1) highlight the state, function, fate, and care of the prepuce in childhood, with focus on the neonate, in Canada; 2) understand the current practice of childhood circumcision in terms of age, indications, performers, techniques, outcomes, and education; and 3) consider ways to sustain a good healthcare professional-parental dialogue for safe practices that are accessible, acceptable, and culturally sensitive in the care of the prepuce.

A literature review of the major databases - PubMed (MEDLINE), EMBASE, The Cochrane Library, CINAHL, Web of Science (WOS), Core Collection, LILAC, Africa Health Line, WHO/UNAIDS, Clinical Trials (www.clinicaltrials.gov), Google Scholar, and grey literature - was done. Keywords included prepuce, diseases of prepuce, prepuce in the neonate, prepuce in the neonate in Canada, male circumcision, childhood male circumcision, neonatal male circumcision in 
Canada, complications of neonatal circumcision in Canada, and circumcision adverse events. Additional information for this review was gathered from clinical practice over the years, information from colleagues, and parents. This was not designed to be a systematic review or meta-analysis of childhood or neonatal circumcision and the foreskin. Rather, the literature search allowed for identification of publications in English from the time of database creation until June 30, 2016, and any updates as of November 30, 2016. There was a preference for articles by Canadian authors; however, other articles in several journals outside Canada were included, when necessary, for better understanding of a global perspective of the subject matter.

\section{Results}

A large amount of published literature in English exists in all the databases searched. Overall, there were relatively few publications on access of care, quality of care, outcomes of care, diseased foreskins, or competencies of circumcision performers. Since the delisting of circumcision from the publicly funded health insurance schemes of all the provinces and territories over the past several decades, the prevalence of circumcision has varied. Some authors reported on the need for proper parental education,,$^{2,3,-7}$ others on the need for quality training and competency of performers of circumcision. ${ }^{8,9}$ Reasons for neonatal /childhood circumcision and contraindications in Canada are shown in Table 1. For potential benefits and risks, refer to Table 2 .

\section{The prepuce: Anatomy, embryology, and function}

Several authors have described the development and anatomy of the prepuce. ${ }^{1,4,10,11}$ In his article, Gairdner quoted the Anatomist/Surgeon Hunter's description of the prepuce appearance in the foetus at eight weeks' gestation as a ring of thickened epithelium that grows distally over the base of the glans penis. ${ }^{1}$ At 12 weeks, the urethra opens ventrally on the shaft of the penis. Arrest of development at this stage results in glandular hypospadias, with the "hooded" prepuce over the dorsal aspect of the glans. By the 16th week, the prepuce has grown distally toward the tip of the glans. Then, the squamous epithelium of the deep surface of the prepuce is continuous with that of the glans. By process of desquamation, the preputial space is formed. At birth, the separation of the foreskin from the glans is not quite complete and the foreskin is non-retractable - a condition several authors refer to as physiological phimosis.

In 1949, Gairdner observed that in a series of 100 newborns, $4 \%$ had full retractable prepuce; in $54 \%$ the glans could be uncovered to show the external urethral meatus; and in the remaining $42 \%$, the tip of the glans could not be uncovered. ${ }^{1}$ He reported that in older age groups, the foreskin remained not retractable by six months in $80 \%$ of boys, by 12 months in $50 \%$, by two years in $20 \%$, and by three years in $10 \%$; the prepuce was still not retractable in $6 \%$ of children aged 5-13 years and could be partially retracted in 14\%. Gairdner recommended that until age three years, a non-retractable prepuce be considered normal and that after this age, attempts be made to retract the prepuce and keep it so for cleanliness. ${ }^{1}$ Other authors have suggested that with watchful waiting and healthy penile hygiene, only $1 \%$ of the boys will have nonretractile prepuce by age 17 years. ${ }^{2,4}$ It has been postulated that with squamous keratinization of the foreskin, accumulation of preputial smegma, and intermittent penile erections, the foreskin in young boys becomes completely retractable. . $^{1,3,7,10,12}$

Gairdner observed that, "it is often stated that the prepuce is a vestigial structure devoid of function." ${ }^{1}$ Several authors have suggested that the prepuce functions to protect the glans penis from injury and irritations from ammoniacal urinary contents. ${ }^{1,3}$ It appears that these protective functions are lost in boys who have fully developed prepuces and still suffer from conditions that the prepuce should have provided a safety "hood" for. The role of the prepuce in erection and sexual satisfaction is uncertain. ${ }^{4,13}$ Overall, it is fair to state the function of the prepuce has not been clearly defined. ${ }^{2,12} \mathrm{~A}$ role of the healthy prepuce not often discussed is the potential benefit for various reconstructive procedures of the urethra.

\section{The fate of the prepuce and choices of care}

The prepuce in the neonate can acquire conditions that affect it and the male genitalia, early or in adulthood. These conditions include phimosis, paraphimosis, balanitis and balanoposthitis, preputial ballooning, and preputial adhesions. Other conditions that may affect or be associated with resident prepuce include dyspareunia, preputial abrasions during intercourse, urinary tract infections, ${ }^{14}$ increased risk of sexually transmitted disease, ${ }^{15-17} \mathrm{HPV}$, AIDS/HIV,, $18-20$ penile cancer, ${ }^{21}$ and balanitis xerotica obliterans (BXO). ${ }^{22,23}$ While some authors advance these conditions to justify a position in favour of routine neonatal circumcision, the balance of evidence in a Canadian context led the Canadian Pediatric Society to maintain the position that circumcision of the newborn should not be routinely performed. ${ }^{6,7}$

In this section of this review, the emphasis is on provision of care for the neonates who are not circumcised and have a normal prepuce for life. The care should be simple, personal, parent-friendly, and easily learned and reproducible. Body and skin care by regular baths with water and soap should be provided by the caregiver/parent until the child is able to do so on his own. There should be no attempts at retracting the prepuce prematurely. When the prepuce becomes retractable, the same routine of hygienic measures should be followed and the child can be taught to gently retract and clean the prepuce daily and as required. 


\begin{tabular}{ll}
\hline $\begin{array}{l}\text { Table 1. Reasons for neonatal/childhood circumcision and } \\
\text { contraindications in Canada }\end{array}$ & \\
\hline $\begin{array}{l}\text { Reasons for neonatal/childhood } \\
\text { circumcision }\end{array}$ & $\begin{array}{l}\text { Contraindications for } \\
\text { circumcision (absolute or } \\
\text { relative) }\end{array}$ \\
\hline - Religious & - Premature baby \\
- Ethnocultural & - Sepsis \\
- Social/personal and family & - Acute balanitis \\
values & - Hypospadias, ambiguous \\
- Medical: Phimosis not & genitalia, hidden or buried \\
responding to topical steroids; & penis \\
phimosis, balanitis xerotica & - Neonatal illness \\
obliterans, paraphimosis, & - Bleeding problems \\
recurrent urinary tract & - Presence of any rash \\
infections, associated & - Outbreak of staphylococcal \\
genitourinary anomalies and & infection in nursery \\
phimosis & - Low Apgar score \\
- Other: Parents want to & - Infant less than 24 hours old \\
prevent disease in adulthood & (it takes time to assess the \\
& infant; have full discussion \\
& with parents) \\
& - Allow baby to acclimatize \\
& to the world outside the \\
& mom's womb (rule out \\
& any contraindications for \\
& circumcision) \\
& Parental wish not to have \\
& their newborn circumcised \\
\hline
\end{tabular}

It has been observed that non-compliance by boys in keeping their prepuce clean and the lack of proper supervision by parents and caregivers can result in failure, making circumcision necessary. ${ }^{24}$

\section{Diseases of the prepuce and associated concerns}

\section{Phimosis}

Phimosis is often defined as a condition where the prepuce cannot be retracted over the glans penis without difficulty and pain. Some authors have classified the condition into physiological phimosis - considered a normal childhood condition where the preputial tissues are normal and nonretractable, and pathological when there is scarring of the distal prepuce with adherence to the glans and narrowing of the preputial opening. ${ }^{10,11}$

Pathological phimosis results from traumatic and inflammatory injuries to the prepuce.

Physiological phimosis is common in boys up to and older than three years and requires no treatment, so long as the tissues are normal and healthy. Several authors have described a number of procedures to relieve pathological phimosis, including manual retraction, topical steroid therapy, dorsal slit, preputioplasty, and circumcision. . $^{3,4,7,10,11,13,24}$

\begin{tabular}{ll} 
Table 2. Outcome: Potential benefits and risks of neonatal \\
and childhood circumcision & \\
\hline Potential benefits & Potential risks \\
\hline - Prevention of phimosis & - Minor bleeding \\
- Prevention of paraphimosis & - Minor local wound infection \\
- Decrease of early child & - Severe infection \\
urinary tract infections & - Urethral and meatal injury \\
- Decrease in urinary tract & - Meatal stenosis \\
infections in boys with & - Unsatisfactory cosmetic \\
genitourinary anomalies & results \\
and recurrent urinary tract & - Death from unrecognized \\
infections & bleeding \\
- Prevention of balanitis & \\
xerotica obliterans & \\
- Decrease acquisition and & \\
transmission of HIV & \\
- Decrease acquisition of & \\
herpes simplex virus & \\
- Decrease acquisition of & \\
human papilloma virus & \\
- Decrease penile cancer risk & \\
- Decrease cervical cancer risk & \\
in female sexual partner & \\
- Decrease chances of having \\
- Dircumcision in adult \\
Decrease costs compared \\
- Simpler adult circumcision \\
\hline
\end{tabular}

\section{Balanitis and balanoposthitis}

Inflammation of the glans penis is balanitis; it is referred to as balanoposthitis when both the glans and the prepuce are involved. It has been reported to occur in $4-11 \%$ of uncircumcised boys. ${ }^{13,25}$ In many cases, no cause can be identified, but retained secretions, bacterial contamination, trauma, contact irritation, and allergy have been noted in the presence of phimosis. ${ }^{13}$

\section{Paraphimosis}

Paraphimosis occurs when the prepuce is retracted proximally and remains entrapped proximal to the corona sulcus. The glans becomes engorged and the prepuce swollen, edematous, and painful. This could happen if boys have been advised by parents or healthcare providers to retract their prepuce for physiological phimosis. Manual decompression, with the help of $2 \%$ xylocaine jelly and a clean gauze can resolve this when the child presents early. In difficult situations, various techniques have been described to resolve paraphimosis., ${ }^{3,11}$

Some authors recommend a dorsal slit or circumcision for paraphimosis to avoid any recurrence, while others state that circumcision is not mandatory. $3,4,7,13$ It is advised that liberal analgesics, both topically and orally, may be necessary for any manipulation to resolve paraphimosis. 


\section{Smegma}

Smegma is a secretion composed of desquamated epithelial cells that accumulates under the prepuce. This is often seen in little boys who do not have fully retractile prepuce. Parents are often concerned that their son may have an infection. Reassurance and advice in healthy penile hygiene is adequate.

\section{Ballooning of the prepuce}

In boys whose prepuce is not fully retractable, ballooning may occur as a result of the tight prepuce. Parents are usually worried that their child may not be able to urinate well. To the contrary, urinary flow almost always goes unimpeded and the ballooning resolves with time. A tinge of topical steroid cream or ointment may be necessary. Parents need reassurance and to be made aware that ballooning of the prepuce is benign. $4,7,10$

\section{Adhesions}

Adhesions are considered by some authors as residue of the fused inner layer of the prepuce and the glans., ${ }^{3,10}$ These are often seen among boys until the teenage years, when they eventually break down as a result of preputial retraction and intermittent erections. Complete resolution by the teenage years is the rule. Good penile hygiene is the best recipe.

\section{Balanitis xerotica obliterans}

Balanitis xerotica obliterans (BXO) or lichen sclerosus (Fig. 1) is an infiltrative lesion of the prepuce that causes pathological phimosis. It is easily recognizable at the tip of the prepuce as a ring of hardened tissue with extensive scarring whitish in colour. Sometimes, it may have variegated skin changes with presence or absence of edema. This condition is rare in boys aged less than five years, but has been reported as a persistent cause of pathological phimosis at puberty. ${ }^{11,12}$ No large cohort, nor case series have been reported in Canada. BXO has been described as an absolute indication for circumcision. ${ }^{22,23}$ These boys present with non-retractable prepuce, dysuria, and occasional obstructive urinary symptoms. Histological examination of the circumcision tissue usually confirms the diagnosis, showing hyperkeratosis with follicular plugging, atrophy of the stratum spongiosum Malpighi with hydropic degeneration of basal cells, lymphedema, hyalinosis and homogenization of collagen in the upper-dermis, and inflammatory infiltration in the mid-dermis. Topical steroid treatment for up to 14 months has proven effective, especially if applied during the active early inflammatory process . ${ }^{4,13,24}$ However, circumcision is the preferred treatment option, as it removes all the affected tissues. Meatotomy or meatoplasty may be required and postoperative topical steroid may be useful to reduce the risk of restenosis. ${ }^{3,4,11}$

\section{Management choices for the prepuce in childhood}

The mainstay of management of the prepuce remains personal hygiene cultured by parental care and diligence by the child. In homes where religious, cultural, ritual affiliations and parental wishes prevail, neonatal circumcision will be procured early in life or as stipulated by the beliefs. For pathological phimosis, topical steroid therapy has been found effective, as reported by several authors, $3,4,7,10,13$ challenging any proposition for routine neonatal circumcision. The other alternative treatment options to circumcision, such as manual retraction, dorsal slit, and preputioplasty are practiced, but have not been reported widely in Canada and globally. Except in special circumstances, these are not recommended, as their results are not long-lasting and may have poor cosmetic and limiting functional outcomes. ${ }^{11}$

\section{Care of the normal prepuce and physiological phimosis in childhood}

As recommended by the guidelines on the care of the normal prepuce and neonatal circumcision in Canadian infants, ${ }^{7}$ examination of the prepuce, urethral meatus, and genitalia should be part of clinical assessment of all newborn baby boys. Continued assessment of the prepuce without forcible retraction of the prepuce should be undertaken during annual physical examination to rule out pathological phimosis and confirm natural prepuce smooth retraction. Some key points from the guideline include:

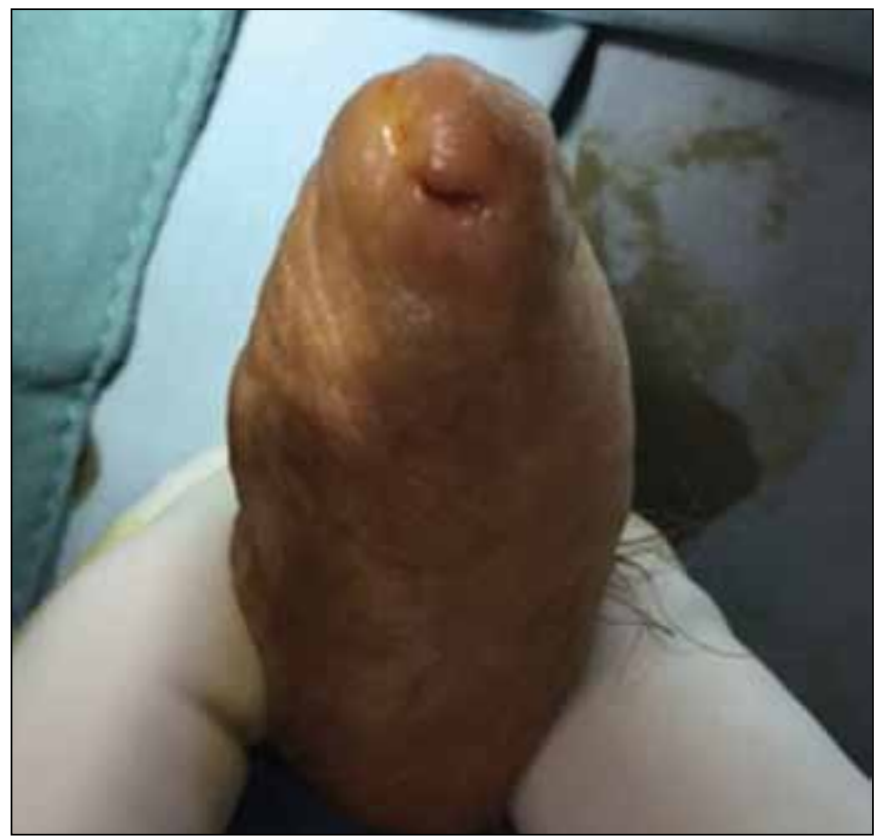

Fig. 1. A case of balanitis xerotica obliterans non-responsive to steroid therapy; required circumcision. 
1. Parents and caregivers should be educated on the care of the prepuce and genitalia of their newborns, ensuring they are familiar with steps of examination, safe-cleaning, and hygiene for the prepuce, with clear instructions to not attempt forceful retraction of the childhood prepuce.

2. Asymptomatic physiological phimosis in a boy should not be an indication for circumcision, nor any physical manipulation.

3. When balanoposthitis and recurrent urinary tract infections (UTIs) occur in the presence of physiological phimosis, antibiotic treatment orally and topically in combination with topical steroid treatment achieve satisfactory outcome with low risk of complications.

4. The choice of topical steroid therapy is individualized and depends on availability, affordability, costs, and response. Moderately low-potency steroids (tramcinolone, clobetasone, hydrocortisone, mometasone) may have the same successful results as a highly potent steroid (bethametasone). ${ }^{4,13}$

\section{Care of the pathological phimosis and the poor compliant boy}

Some important factors in the medical management of nonretractile prepuce include parental economic and social status, motivation to follow advice, and the ability to remain patient and supportive of the child as he learns about self-care.

Warner and Strashin have noted in their review the potential for non-compliance to the rules of good hygiene and proper application of topical creams to lead to poor response and early referral for circumcision. ${ }^{24}$ Therefore, careful selection of patients to ensure compliance, good demonstration of gentle retraction of prepuce, application of the steroid cream, and continued intermittent retraction after initial success will assure ongoing success with topical steroid therapy.

In a survey of 34 Canadian pediatric urologists, Metcalfe and Elyas found that there was similarity in their adopting a conservative management of common prepuce issues, including circumcision for BXO. ${ }^{22}$ Failure of topical steroid therapy and hygiene in a boy and/or poor compliance warrants circumcision, with full disclosure of risks, benefits, potential complications, alternatives, and costs to the family.

\section{To keep or not to keep the prepuce: The circumcision factor}

Male circumcision is the surgical removal of the prepuce. It is widely considered to be one of the first surgical incisions made by man. The word "circumcision" is derived from the
Latin word "circumdidere" (meaning "to cut around"). It is practiced in different parts of the world for cultural, religious, social, or prophylactic reasons (non-therapeutic), or to treat an underlying disease process (therapeutic).

Neonatal and childhood circumcision in Canada is commonly practiced for parental preferences and/or for social, cultural, hygiene-specific purposes and for pathological conditions that do not respond to non-invasive and medical techniques (Table 2). Prevalence rates of neonatal circumcision in Canada have fluctuated over the years, decreasing since the position statement of the Canadian Pediatric Society in $1975^{23}$ and delisting of the procedure by publicly funded insurance of the provinces and territories across the country in past four decades. ${ }^{26}$

Hospital-derived data reported for neonatal circumcision rates in 1970-1971 varied from $42 \%$ in Nova Scotia to $67 \%$ in Alberta; the prevalence rates went down during the 1970s, with the lowest rate of $13 \%$ in Quebec and $22 \%$ in Nova Scotia in 1976. ${ }^{26}$ The policy statements from the American Academy of Pediatrics and the Canadian Pediatric Society likely influenced this decline. ${ }^{5-7,27} \mathrm{~A}$ more recent study of 69100 neonates in Ontario born in 1993-1994, found a prevalence rate of $44 \%{ }^{26}$ there was evidence of declining hospital-based circumcision rates among male infants $<28$ days old in Ontario during the 1990s (in-hospital prevalence of 39\% from 1989-1992 and 30\% from 1994-199526). In 2007, estimation of number of Canadian males aged 15 or older circumcised for non-religious reason was reported as $30 \%{ }^{28}$ and may have changed slightly since then.

\section{Preferred age for childhood circumcision}

The neonatal period is the most popular and preferred age for childhood circumcision due to religious, social, cultural/ethnic reasons. The procedure has been described as simple by many authors. ${ }^{2,3,6,7,11}$ It is relatively quick and safe when performed under aseptic conditions by a trained, skilled professional. Complication rates are usually minor $(0.2-2.0 \%)$. ${ }^{8,29-32}$

Parents are aware of the requirements and rituals in their various ethnic groups and religions are often anxious to secure access for an acceptable facility and date for the circumcision.

Children older than 28 days require full anesthesia and there may be limitations in supply of trained and willing anesthetists and equipment in community or rural hospitals. In some jurisdictions and practices, infant male circumcisions are carried out with local anesthesia.

Cost of the circumcision is cheaper when the child is less than 28 days old. After the neonatal period, the cost of circumcision increases astronomically.

It is generally acknowledged that neonatal circumcision carries lower risks and costs than later childhood circumcision, considered to be harder on the child and to carry the 
potential of increased post-procedural pain and complication rates up to $6 \% .^{29,32}$

\section{Who performs circumcision in Canada?}

In Canada, most neonatal male circumcisions are performed by medical practitioners, including obstetricians, neonatologists, pediatricians, general practitioners, general surgeons, pediatric surgeons, urologists and, rarely, pediatric urologists, who generally serve as consulting physicians in cases of circumcision-related complications. ${ }^{6,7,22,29,33}$

There are also some skilled traditional circumcisers (e.g., Mohel) who have code of ethics and conduct and practice under hygienic environments. In southwestern Ontario, neonatal circumcisions are performed by family doctors and pediatricians $(85 \%)$ and pediatric general surgeons and urologists $(15 \%){ }^{9}$

\section{Techniques and devices for childhood/neonatal circumcision}

Details of techniques and devices used for childhood male circumcision are varied and widely available. For neonatal circumcision, devices such as the Gomco $^{\circledR}$ (Yellen clamp), Plastibell (Hollister Inc. Chicago, IL, U.S.), and the Mogen clamp $(29,30)$ are the three more popular devices available in the U.S. and Canada. ${ }^{29,34}$ They are also widely used all over the world.

There are newer devices recently approved by the World Health Organization (WHO). ${ }^{29}$ The Gomco ${ }^{\circledR}$ device has a metal cone that protects the glans and a clamp that holds the prepuce tightly in position and secures hemostasis before the incision is made. This device may result in increased risk for bleeding and injury to the glans. The Plastibell, on the other hand, comprises the use of plastic cap and ligatures rather than a clamp. The distal prepuce is excised and the distal part of the cap removed so that part of the prepuce strangulated by ligature will ring of the cap within 10 days, necrosis, and fall off. This device is associated with a greater risk of infection and retained device if the size is not right, as it must be left in place for at least one week. The scalpel and scissors technique has stood the test of time; the dorsal slit and the sleeve techniques have been used by surgeons and "circumcisionists" with very good results over the years. Newer devices are available or in the process of development following guidelines from the WHO. ${ }^{29,34}$

\section{Anesthesia for childhood circumcision}

Good and safe anesthesia are highly recommended and have made the neonatal and childhood circumcision safer, with negligible serious adverse effects and complications. Any programs or professionals wishing to establish a neonatal and childhood circumcision service need to familiarize themselves with the WHO/UNAIDS handbook that has been developed for circumcisions under local anesthesia. ${ }^{29}$ For children older than 28 days, general anesthesia is recommended. Such boys may have to wait until they are about 18 months or older to have their circumcisions done. As the procedure is considerably more expensive at this age, most parents eagerly strive to have their newborns circumcised within the first two weeks of life.

\section{Family/parental counselling and consent}

There is a great need for physicians and healthcare providers to be very knowledgeable about the care of the neonatal/ childhood prepuce and, in turn, to better inform parents about the care of their newborn. In this age of digital information technology and social media, parents are likely to come to healthcare providers with valid questions. A careful, detailed, unbiased dialogue about the known facts on the choices of care for the prepuce, including circumcision, should be provided. The parents can then weigh the specific risks and benefits in the context of their personal values, religious and ethno-cultural beliefs, and make their decision.

\section{Pre- and postoperative care and followup}

In the literature reviewed, there were no standardized followup patterns, nor classification of complications and outcome measures. It is necessary to provide appropriate pre- and postoperative care information and care plan. The healthcare provider and the parents need to be aware of the contraindications to circumcision. In one of the papers, the authors were surprised to learn that some of the physicians who performed circumcisions were not familiar with some of the contraindications. ${ }^{19,33}$ Though considered an easy and simple operation, it is imperative that parents are provided adequate information on care plan before and after the operation. Video, written, verbal communication or social media could be easy portals to make this possible. In the early postoperative period, when there is a small risk of bleeding, help should be easy to reach to avoid unnecessary morbidity, as the child with a small blood volume can suffer.

\section{Complications}

The prevalence of neonatal circumcision complications is reported to be low and easily treated, but there are no standard methods that have been adopted. Most reports have biases that are related to retrospective studies and the potential deficiencies noted when working with hospital records (i.e., each hospital reporting severity of complications differently or not reporting at all). In their 1981 review, Warner and Strashin noted ranges from as low as $0.06 \%$ to a high of $55 \%$ reported by Kingston General Hospital. ${ }^{24,35}$ This seems to be the trend in the literature; several large series from different parts of the 
world recorded similar low complication rates: In Israel, Ben Chaim et al report 1.2\%; ${ }^{32}$ in Nigeria, Biuko et al report 1.1\% ${ }^{30}$ and in the U.S., El-Bcheraoul et al report $0.5 \%{ }^{8}$

In their study, El-Bcheraoul et al concluded that male circumcision had a low incidence of adverse events overall, especially if the procedure was performed during the first year of life, but rose 10-20-fold when performed after infancy. ${ }^{8}$ In 2010, Weiss et al published a systematic review and meta-analysis of 52 studies from 21 countries, with Arabic literature included. ${ }^{31}$ They included 16 prospective studies that evaluated complications following neonatal and infant circumcision. Most studies reported no severe adverse events, but two studies reported a $2 \%$ rate of severe adverse events. The median frequency of any complications was $1.5 \%$ (range $0-16 \%$ ). Childhood circumcision by medical providers tended to be associated with more complications (median frequency $6 \%$; range $2-14 \%$ ) than for neonates and infants. Traditional circumcision as a rite of passage is associated with greater risks, more severe complications than medical circumcision or traditional circumcision among neonates. The authors concluded that the studies reviewed report few severe complications following circumcision, but mild to moderate complications do occur, especially when circumcision is performed in older age group and/or by inexperienced providers in non-sterile conditions.

Childhood circumcision will continue to be practiced for cultural, ethnic, and medical purposes, as well as a longterm HIV/sexually-transmitted infection preventive strategy. No recent large cohort studies describing prevalence of post-neonatal circumcision complications in Canada was identified. Based on the policy statement by the Canadian Pediatric Society in 2012, ${ }^{6}$ and reaffirmed in $2015,{ }^{7}$ neonatal circumcision services are safe with low early and late complications and adverse events. ${ }^{11,31,32,35}$

\section{Teaching the techniques of neonatal/childhood circumcision}

Until about 10 years ago when the WHO/UNAIDS produced a handbook on the practice of circumcision, formal structured teaching on techniques, safe practices, indications, and contraindications was rare. In Canada, DeMaria et al, in a survey of community physicians performing neonatal circumcisions, found out that most of these physicians received informal and unstructured training. ${ }^{9}$ The authors developed and organized an effective workshop with six-month postworkshop assessment of attendees. The goal was to improve the outcome and reduce the complications of neonatal circumcision. However, the authors did not comment on how to sustain and strengthen further continued medical education, nor any plans to promote their website. In this era of competency-based medical education, teaching and performance of neonatal/childhood circumcision may result in satisfactory outcomes, further reducing the complication rates. ${ }^{29,33}$

\section{Overview and recommendations}

In Canada, we need more prospective studies with monitoring of risks and benefits. Such studies should be able to document complications with standardized definitions of minor, moderate, and severe, as well as protocols for early and late followup. We need to understand the risks associated with different techniques and age at circumcision, as the literature suggests that procedures are tailored to specific age groups.

There should be educational programs offered on the care of the healthy prepuce, with teaching and counselling tools on simple penile hygiene and healthy reproductive and sexual education. There is a clear need to improve the safety of neonatal/childhood circumcision at all ages through improved training or retraining of both traditional and medical providers and provision of necessary and adequate equipment for safe circumcision. If not already in place, guidelines for safe neonatal/childhood circumcision should be developed. Good communication between the formal and informal circumcision providers could assist in avoiding unnecessary morbidity.

Local anesthesia for neonatal circumcision is recommended by WHO/UNAIDS. ${ }^{28,29}$ For older children (more than 28 days old), general anesthesia is required and the anesthetist may require that the parents wait until safe anesthesia is right. Anaesthesia risk is lower after three months and should be performed at a pediatric centre before one year.

Circumcision is undertaken by a range of providers, with the choice of provider depending on family, religious, and ethno-cultural background, cost of procedure, availability, accessibility, and perception of service quality.

As a traditional religious or sociocultural practice, neonatal/childhood circumcision is likely to remain prevalent around the world, even in countries like Canada where there has been a decline over the past four decades. Every effort should be made to ensure that the procedure is performed safely by trained and experienced providers equipped adequately in a safe and aseptic environment.

\section{Who is the piper? Who pays the piper? Who dictates the tune?}

Public health considerations of prepuce health are multifaceted. There is the safety and integrity of a prepuce free of disease and irritation, and the safety of circumcision in and of itself. There are costs involved, no matter how small. There is personal responsibility for customized care 
of a healthy, uncircumcised or circumcised penis. Legal issues and ethical boundaries permeate the discourse on circumcision and the status of the intact prepuce. Health consequences of procedures and practices in reproductive and sexual life have been widely studied and published. In Canada, cultural acceptability and sensitivity play an important role in healthcare choices for the prepuce.

From the review of the literature, it appears that most Canadians are living with their prepuce. We do not have the accurate information on how many have issues or irritations with their prepuce and how bothersome these may be. Healthy lifestyles, reproductive and sexual health education currently available, and appropriate quality improvement should serve the individual to maintain the "hood" for life. Perhaps we need more studies to understand all aspects of the well-being of the prepuce.

For the $30-32 \%$ of Canadians who may not retain their prepuces for life as a result of substitute decision or consent for circumcision, adequate provision for good, affordable, accessible, culturally sensitive, non-discriminatory, and safe care should be available.

Ethical and legal issues about the care of the neonatal prepuce and circumcision are beyond the scope of this review. Readers are best advised to consult the July 2013 issue of the Journal of Medical Ethics, which is devoted fully to the topic. ${ }^{36}$

Competing interests: The author reports no competing personal or financial interests.

Acknowledgments: The staff and professional development team at the Richmond Hill Urology Practice and Prostate Institute were very helpful in the literature search and contacting authors of articles when necessary.

This paper has been peer-reviewed.

\section{References}

1. Gairdner D. The fate of the foreskin: A study of circumcision. BMJ 1949;2:1433-7. https://doi.org/10.1136/bmi.2.4642.1433

2. Oster J. The further fate of the foreskin. Arch Dis Child 1968;43:200-3.

3. Shahid SK. Phimosis in children. ISRN Urology 2012;2012:707329. https://doi.org/10.5402/2012/707329

4. McGregor TB, Pike JG, Leonard MP. Pathologic and physiologic phimosis: Approach to the phimotic foreskin. Can Fam Physician 2007:53:445-8.

5. American Academy of Pediatrics, Task Force on Circumcision. Circumcision policy statement. Pediatrics 1999;103:686-93. https://doi.org/10.1542/peds.103.3.686

6. Task Force on Circumcision. Male circumcision. Pediatrics 2012;130:e756-e85 https://doi.org/10.1542/peds.2012-1990

7. Sorokan ST, Finlay JC, Jeffries AL. Newborn male circumcision. Paediatr Child Health 2015; 20;311-5.

8. El-Bcheraoul $C$, Zhang $X$, Cooper $C S$, et al. Rates of adverse events associated with male circumcision in U.S. medical settings, 2001 to 2010. JAMA Pediatr 2014;168:625-34. https://doi.org/10.1001/ jamapediatrics.2013.5414

9. DeMaria J, Abdulla A, Pemberton J, et al. Are physicians performing neonatal circumcisions well-trained? Can Urol Assoc J 2013;7:260-4. https://doi.org/10.5489/cuai.200
10. Dobanovacki D, Prostran B, Sarac D, et al. Prepuce in boys and adolescents: What, when, and how? Med Pregl 2012;65:295-300. https://doi.org/10.2298/MPNS1208295D

11. Hayashi Y, Kojima Y, Mizuno K, et al. Phimosis, paraphimosis, and circumcision. ScientificWorldJournal 2011;11:289-301. https://doi.org/10.1100/tsw.2011.31

12. Morris $B J$, Wamal RG, Henebeng EB, et al. Estimation of country-specific and global prevalence of male circumcision. Popul Health Metr 2016;14:11. https://doi.org/10.1186/s12963-016-0080-6

13. Van Howe RS. Cost-effective treatment of phimosis. Paediatrics 1998;102:E43. https://doi.org/10.1542/peds.102.4.e43

14. Herzog LW. Urinary tract infections and circumcision. A case-control study. A J Dis Child 1989;143:348-50.

15. Tobian AAR, Gray RH, Quin TC. Male circumcision for prevention of acquisition and transmission of sexually transmitted infections: The case for neonatal circumcision. Arch Pediatr Adolesc Med 2010;164:78-84. https://doi.org/10.1001/archpediatrics.2009.232

16. Van Howe RS. Sexually transmitted infections and male circumcision: A systematic review and metaanalysis. ISRN Urol 2013;2013:109846. https://doi.org/10.1155/2013/109846

17. Mehta $S$, Moses $S$, Agot $K$, et al. Adult male circumcision does not reduce the risk of incident Neisseria gonorhoeae, Chlamydia trachomatis, or Trichomonas vaginalis infection: Results from a randomized, controlled trial in Kenya. J Infect Dis 2009;8: 370-8. https://doi.org/10.1086/6000743

18. Bailey R, Moses $S$, Parker $C$, et al. Male circumcision for HIV prevention in young men in Kisumu, Kenya: A randomized, controlled trial. Lancet 2007;369:643-56. https://doi.org/10.1016/S0140$6736(07) 60312-2$

19. Auvert B, Sobngwi-Tambekou J, Cutler E, et al. Effect of male circumcision on the prevalence of high-risk human papilloma virus in young men: Results of a randomized, controlled trials conducted in Orange Farm, South Africa. J Infect Dis 2009;1:14-9. https://doi.org/10.1086/595566

20. Weiss HA, Thomas SL, Munabi SK, et al. Male circumcision and risk of syphilis, chancroid, and genital herpes: A systematic review and meta-analysis. Sex Transm Infect 2006;82:101-10. https://doi.org/10.1136/sti.2005.017442

21. Larke N, Thomas SL, dos Santos Silva I, et al. Male circumcision and penile cancer: A systematic review and meta-analysis. Cancer Causes Control 2011; 22:1097-110. https://doi.org/10.1007/ s10552-011-9785-9

22. Metcalfe P, Elyas R. Foreskin mamagement: A survey of Canadian pediatric urologists. Can Fam Physician 2010;56: e290-5.

23. Meuli M, Briner J, Hanimann B, et al. Lichen sclerosus et atrophicus causing phimosis in boys: A prospective study with a 5-year followup after complete circumcision. J Urol 1994;152:987-9.

24. Warner E, Strashin E. Benefits and risks of circumcision. CMAJ 1981;125: 967-76.

25. Fetus and Newborn Committee. FN-75-01: Circumcision in the newborn period. CPS News Bull Suppl 1975;8:1-2.

26. To T, et al. Pediatric health service utilization: Circumcision. In: Goel V et al., eds., Patterns of healthcare in Ontario: The ICES practice atlas, Ottawa. Canadian Medical Association, 1996: 294-6.

27. American Academy of Pediatrics, Committee of the Fetus and Newborn. Standards and recommendation for hospital care of the newborn infants. Evanston, Academy of Pediatrics 1971:110.

28. WHO/UNAIDS (2007-2008). Male circumcision: Global trends and determinants of prevalence, safety, and acceptability. http://www.unaids.org/sites/default/files/media_asset/ic1360_male_circumcision_en_2.pdf. Accessed January 18, 2017.

29. WHO/UNAIDS 2010 Neonatal infant, child circumcision: A review. http://www.who.int/hiv/pub/ malecircumcision/neonatal_child_MC_UNAIDS.pdf?ua=1. Accessed January 18, 2017.

30. Jimoh BM, Odunayo IS, Chinwe I, et al. Plastibell circumcision of 2276 male infants: A multicentre study. Pan Afr Med J 2016;23:35. https://doi.org/10.11604/pami.2016.23.35.7841

31. Weiss HA, Larke N, Halperin D, et al. Complications in male neonates, infants, and children BMC Urol 2010;10:2. https://doi.org/10.1186/1471-2490-10-2

32. Ben Chaim J, Levin PM, Binyamin J. Complications of circumcision in Israel: A one-year multicentre survey. Isr Med Assoc J 2005;7:368-70.

33. Metcalfe P. Teaching neonatal circumcision. Can Urol Assoc J 2013;7:265. https://doi.org/10.5489/ cuaj. 1523

34. Kurtis PS, DeSilva HN, Bernstein BA, et al. A comparison of the Mogen and Gomco clamps in combination with dorsal penile nerve block in minimizing the pain of neonatal circumcision. Paediatric 1999;103:e2.

35. Patel H. The problem of routine circumcision. Can Med Assoc J 1966;95:576-81.

36. Foddy B. Medical, religious, and social reasons for and against an ancient rite. J Med Ethics 2013;39:415. https://doi.org/10.1136/medethics-2013-101605

Correspondence: Dr. Emmanuel 0. Abara, Clinical Sciences Division, Northern Ontario Medical School, Sudbury/Thunder Bay, ON, Canada; rhuppi@rogers.com 